\title{
Long non-coding RNA AC023794.4-201 exerts a tumor-suppressive function in laryngeal squamous cell cancer and may serve as a potential prognostic biomarker
}

\author{
ZHISEN SHEN $^{1}$, JIE YUAN ${ }^{1,2}$, QIAOLING TONG ${ }^{3}$, WENJUAN HAO ${ }^{1}$, HONGXIA DENG $^{1}$, \\ QUN LI ${ }^{1}$, CHONGCHANG ZHOU ${ }^{1}$, YAN HU ${ }^{1,2}$ and JIE XU ${ }^{1,2}$ \\ ${ }^{1}$ Department of Otorhinolaryngology and Head and Neck Surgery, Li Huili Hospital Affiliated to Ningbo University, \\ Ningbo, Zhejiang 315040; ${ }^{2}$ Department of Otorhinolaryngology, Medical School of Ningbo University, \\ Ningbo, Zhejiang 315211 ; $^{3}$ Department of Otorhinolaryngology and Head and Neck Surgery, \\ Ningbo No. 2 Hospital, Ningbo, Zhejiang 315010, P.R. China
}

Received July 28, 2019; Accepted February 20, 2020

DOI: 10.3892/ol.2020.11595

\begin{abstract}
After the expression level of lncRNA AC023794.4-201 was upregulated in 2 laryngeal squamous cell carcinoma (LSCC) cell lines (AMC-HN-8 and TU-212) and LSCC xenografts, the biological function of lncRNA AC023794.4-201 in LSCC was further investigated using in vitro and in vivo experiments, such as cell function experiments and nude mice transplantation. In our previous study, it was demonstrated that the expression level of the long non-coding RNA (lncRNA) AC023794.4-201 were decreased in laryngeal squamous cell carcinoma, particularly in cases of LSCC with lymphatic metastasis. Moreover, low expression levels of AC023794.4-201 were revealed to be an adverse prognostic factor for patients with LSCC. In the present study, lentiviruses were used to overexpress AC023794.4-201 before a series of cell function assays were performed and a xenograft nude mouse model was constructed, in order to further investigate the functions of AC023794.4-201 in LSCC. AC023794.4-201 inhibited the proliferation and the cloning capacity of LSCC cells compared with the negative control group as indicated by real-time cell analysis and the plate colony formation assay. Flow cytometry and transwell migration assays demonstrated that AC023794.4-201 inhibited the migration, induced cell cycle arrest and increased the apoptotic rate of LSCC cells. The results of the in vivo studies demonstrated that AC023794.4-201 significantly inhibited the growth of LSCC xenografts, and promoted apoptosis. In
\end{abstract}

Correspondence to: Professor Zhisen Shen, Department of Otorhinolaryngology and Head and Neck Surgery, Li Huili Hospital Affiliated to Ningbo University, 57 Xingning Road, Ningbo, Zhejiang 315040, P.R. China

E-mail: szs7216@163.com

Key words: long non-coding RNA, AC023794.4-201, laryngeal squamous cell carcinoma, prognostic biomarker conclusion, the findings of the present study suggested that AC023794.4-201 may exert tumor-suppressive functions in the progression of LSCC and may serve as a potential prognostic biomarker for LSCC.

\section{Introduction}

Laryngeal squamous cell carcinoma (LSCC), a common malignant tumor, is closely associated with tobacco usage (1). In 2018, there were an estimated 13,150 new cases of LSCC in the USA alone (2). The symptoms of early-stage LSCC including, mild hoarseness, dysphonia, a cough and a hypopharyngeal foreign body sensation, are atypical and easy to ignore. Thus, in clinical practice, LSCC is most typically diagnosed via a pathological biopsy using a laryngoscope or imaging techniques (3). The survival rate of patients with early-stage LSCC is $\sim 80-90 \%$, which is significantly higher compared with patients with advanced LSCC $(4,5)$; however, at the time of diagnosis, the majority of patients are already in the advanced stages of LSCC or exhibit neck lymph node metastasis, demonstrating a survival rate of $50 \%$ (4). Unfortunately, to date, there are no specific diagnostic markers or prognostic/predictive markers for early-stage LSCC. Thus, the requirement to identify effective prognostic biomarkers for patients with LSCC remains crucial.

The pathogenesis of LSCC is highly complex and involves not only environmental factors but also genetic dysregulation $(3,6)$. Long non-coding RNAs (lncRNAs) are a class of RNAs of $>200$ nucleotides in length that have no protein coding ability (7). In fact, the Genome Project reported that $>98 \%$ of genes in the entire genome are non-protein coding genes that are transcribed into non-coding RNA (ncRNA), of which $76 \%$ of ncRNAs are transcribed into IncRNAs $(8,9)$.

IncRNAs have attracted increasing attention in the previous few years in the field of cancer research and have been proven to serve a core regulatory role in protein synthesis (10). IncRNAs also influence genomic loci imprinting, chromosomal conformational shaping, allosterically regulating enzyme activity and numerous other important biological activities, including 
coordinating the cell status and differentiation $(7,11,12)$. Notably, the upregulation, deletion and mutation of lncRNA genes has been implicated in numerous types of human disease, especially cancer (13).

In our previous study, the expression levels of AC023794.4-201 were analyzed in LSCC tissues, adjacent non-cancerous tissues and lymphatic metastatic tissues; AC023794.4-201 expression levels were decreased in the carcinoma tissues of patients with LSCC with low differentiation, high $\mathrm{T}$ staging and cervical lymph node metastasis compared with other tissue samples (14). In addition, low AC023794.4-201 expression levels were discovered to be a poor prognostic factor for LSCC (14). In the present study, cell function assays were performed and a xenograft nude mouse model was constructed to further investigate the functions of AC023794.4-201 in LSCC.

\section{Materials and methods}

Cell culture. Two human LSCC cell lines, TU-212 and AMC-HN8, were obtained from Jennio Biotech Co., Ltd. and the BeNa Culture Collection, respectively. The cell lines were authenticated by short tandem repeat profiling and tested for mycoplasma. TU-212 cells were cultured in RPMI-1640 medium (HyClone; GE Healthcare Life Sciences), supplemented with $10 \%$ FCS (PAN-Biotech $\mathrm{GmbH}$ ), and AMC-HN8 cells were cultured in high-glucose DMEM (HyClone; GE Healthcare Life Sciences), supplemented with 10\% FCS (PAN Biotech UK, Ltd.). Both cell lines were cultured in an incubator at $37^{\circ} \mathrm{C}$ with $5 \% \mathrm{CO}_{2}$.

Lentiviral transfection. To increase the expression levels of AC023794.4-201 in LSCC cells, AC023794.4-201 overexpression lentiviruses and empty lentiviruses vector (LV5-EF1a-GFP/Puro) were purchased from Shanghai GenePharma Co., Ltd. to transfect LSCC cells. Vectors of lentiviruses are plasmids. According to the GenePharma lentivirus operation manual, the cells $\left(3 \times 10^{5}\right.$ per hole plate $)$ were transfected with the lentiviruses $\left(5 \times 10^{8} \mathrm{TU} / \mathrm{ml}\right)$ in a 6 -well plate in their respective medium, supplemented with $10 \%$ FCS. Following $24 \mathrm{~h}$ of incubation, the original medium containing the lentiviruses was removed and replaced with Opti-MEM I reduced serum medium (Gibco; Thermo Fisher Scientific, Inc.). LSCC cells overexpressing AC023794.4-201 were named LV5-AC023794.4-201-transfected cells and empty lentivirus-transfected LSCC cells carrying no target gene were named LV5-negative control (NC)-transfected cells. A group of cells not transfected with lentivirus was set as the blank group.

Quantitative real-time reverse transcription-polymerase chain reaction $(q R T-P C R)$. Total RNA was extracted from experimental and control cells of two cell lines (AMC-HN8 and TU-212) and xenograft tumor samples using TRIzol ${ }^{\circledR}$ reagent (Thermo Fisher Scientific Inc.). The concentration and purity of the total RNA was measured using a SmartSpec Plus spectrophotometer (Bio-Rad, Laboratories); when the A260/A280 value was between 1.8 and 2.0, the purity of the total extracted RNA was considered acceptable. Total RNA from each sample was reverse transcribed into cDNA using the GoScript ${ }^{\mathrm{TM}}$ Reverse Transcription system kit (Promega Corporation) according to the manufacturer's instructions. qPCR was subsequently performed using a Mx3005P fluorescence quantitative PCR instrument (Stratagene; Agilent Technologies, Inc.), according to the manufacturer's protocol, and a GoTaq ${ }^{\circledR}$ qPCR Master mix kit (Promega Corporation). The primers used for the PCR were synthesized by Sangon Biotech Co., Ltd. and the sequences are presented in Table I. The following thermocycling conditions were used for the qPCR: Initial denaturation at $95^{\circ} \mathrm{C}$ for $5 \mathrm{~min}$; and 40 cycles of denaturation at $94^{\circ} \mathrm{C}$ for $15 \mathrm{sec}$, annealing at $55^{\circ} \mathrm{C}$ for $30 \mathrm{sec}$ and elongation at $72^{\circ} \mathrm{C}$ for $30 \mathrm{sec}$. Expression levels were determined using the $2^{-\Delta \Delta \mathrm{cq}}$ method (15) and normalized to GAPDH, the internal reference gene. All samples were tested in triplicate and the results were averaged.

Cell proliferation assay. The proliferation of LSCC cells was analyzed using a real-time cell analyzer (RTCA) assay and the cell index (CI) was regarded as an indicator of cell proliferation. Briefly, $24 \mathrm{~h}$ after transfection, $5 \times 10^{5}$ cells $/ \mathrm{ml}$ cultured in normal media were plated into an E-plate 96, which was subsequently placed in an RTCA (Agilent Technologies, Inc.) to monitor cell proliferation. Proliferation was monitored every $15 \mathrm{~min}$ for a total of $96 \mathrm{~h}$.

Colony formation assay. A colony formation assay was also performed to investigate cell proliferation. Following treatment for $24 \mathrm{~h}$, the cells were dissociated with EDTA containing $0.25 \%$ trypsin and 500 cells/well were plated into a 6-well plate. The 6-well plate was incubated in a cell culture incubator at $37^{\circ} \mathrm{C}$ for 2 weeks. Following the incubation at room temperature the colonies were fixed with $4 \%$ paraformaldehyde for $15 \mathrm{~min}$ and stained with a $0.1 \%$ crystal violet for 20 min staining solution. The stained colonies were manually counted by eye. Each clone contained $>50$ cells, ranging in size from $0.3-1.0 \mathrm{~mm}$.

Flow cytometric analysis of the cell cycle. Cells were serum-starved for $24 \mathrm{~h}$ to synchronize the cells in the $\mathrm{G}_{0}$ phase. The cells were then transfected and collected $24 \mathrm{~h}$ following transfection. Subsequently, cells were rinsed with PBS and fixed in $70 \%$ ethanol at $-20^{\circ} \mathrm{C}$ for $24 \mathrm{~h}$. After fixation, pre-cooled $\left(4^{\circ} \mathrm{C}\right) \mathrm{PBS}$ was added to rehydrate the cells for $15 \mathrm{~min}$. According to the manufacturer's protocol, $500 \mu 1$ propidium iodide (PI)/RNase cell cycle staining kit reagent [Hangzhou Multi Sciences (Lianke) Biotech Co., Ltd.] was added and incubated at $37^{\circ} \mathrm{C}$ for $30 \mathrm{~min}$ in the dark. The cell cycle distribution of the stained cells was determined using a BD FACSCalibur ${ }^{\mathrm{TM}}$ flow cytometer (BD Biosciences).

Flow cytometric analysis of apoptosis. Software FlowJo ${ }^{\circledR}$ v.10.5.2 (FlowJo LLC) was used to analyze results. Transfected cells were dissociated with $0.25 \%$ trypsin (EDTA-free) and washed with PBS. According to the manufacturer's instructions, the resuspended cells $\left(1 \times 10^{6} / \mathrm{ml}\right)$ were stained with Annexin V-FITC and PI using the Annexin V-FITC/PI cell apoptosis staining kit (BD Biosciences). Following $15 \mathrm{~min}$ of incubation at room temperature, apoptotic cells were analyzed using a BD FACSCalibur ${ }^{\mathrm{TM}}$ flow cytometer (BD Biosciences). 
Table I. Primer sequences of AC023794.4-201 and GAPDH used for reverse transcription-quantitative PCR.

\begin{tabular}{ll}
\hline Gene & \multicolumn{1}{c}{ Primer sequence $\left(5^{\prime} \rightarrow 3^{\prime}\right)$} \\
\hline AC023794.4-201 & F: AGAAGAGGGGGAAAAAAG \\
& GATGAAG \\
RA: CGATGGTTAGGGGTGGGAAAGTC \\
F: ACCCACTCCTCCACCTTTGAC \\
& R: TGTTGCTGTAGCCAAATTCGTT
\end{tabular}

F, forward; R, reverse.

Transwell assay. Cells $\left(4 \times 10^{5} / \mathrm{ml}\right)$ were plated in the upper chambers of Transwell plates in serum-free culture medium specific for each cell line. Cell culture medium for each cell line, supplemented with $10 \%$ FCS, was plated in the lower chambers. Following $24 \mathrm{~h}$ of incubation at $37^{\circ} \mathrm{C}$, the non-migratory cells remaining on the membrane were removed. The migratory cells in the lower chamber were stained with $4 \%$ methanol and $0.1 \%$ crystal violet (Sigma-Aldrich; Merck KGaA) at room temperature for $10 \mathrm{~min}$. Stained cells were visualized and imaged using a binocular inverted microscope (Olympus, Japan; Magnification, x40).

Xenograft nude mouse model experiment. All animal studies were approved by and strictly followed the guidelines of the Animal Ethics and Welfare Committee of Ningbo University (approval no. AWEC-2015-10). A total of 32 BALB/c nude mice (age, 4 weeks; sex, male; weight, 14-16 g) were provided by Shanghai SIPPR-Bk Lab Animal Co., Ltd. All nude mice were raised and tested at the Ningbo University Laboratory Animal Center at room temperature, $25^{\circ} \mathrm{C}$ and humidity $70 \%$. The mice had sterile water and food sterilized by radiation and their cage liner was replaced every 2 days. A cell suspension with a cell concentration of $5 \times 10^{7} / \mathrm{ml}$ was made by mixing the AMC-HN-8 cells and the matrigel (BD Biosciences). Each nude mouse armpit was subcutaneously inoculated with $0.2 \mathrm{ml}$ AMC-HN-8 cell suspension that is mentioned previously. Ten days after inoculation, the mice were stochastically assigned to 4 groups ( 8 mice/group): i) Blank control group (no treatment); ii) LV5-NC group, which were injected in xenograft with $50 \mu \mathrm{l} \mathrm{LV5-NC} \mathrm{twice/week} \mathrm{for} 28$ days; iii) low-dose group, which were injected in xenograft with $5 \times 10^{7} \mathrm{TU} / \mathrm{ml}$ LV5-AC023794.4-201 twice/week for 28 days; and iv) high-dose group, which were injected in xenograft with $5 \times 10^{8} \mathrm{TU} / \mathrm{ml}$ LV5-AC023794.4-201 twice/week for 28 days. The weight, maximum length and maximum width of the tumors were measured twice/week. The maximum length(a) and maximum width(b) of the tumors were measured with a vernier caliper. Tumor volume $\left(\mathrm{mm}^{3}\right)$ is equal to $1 / 2 \mathrm{ab}^{2}$. The maximum diameter and volume of a single tumor was $18.20 \mathrm{~mm}$ and $1,114.76 \mathrm{~mm}^{3}$, respectively. On day 29 , the first day of drug withdrawal, the mice were sacrificed with an intraperitoneal injection of $200 \mathrm{mg} / \mathrm{kg}$ pentobarbital and the tumors were harvested. Tumor specimens were fixed for histopathological examination and transmission electron microscopy (TEM).

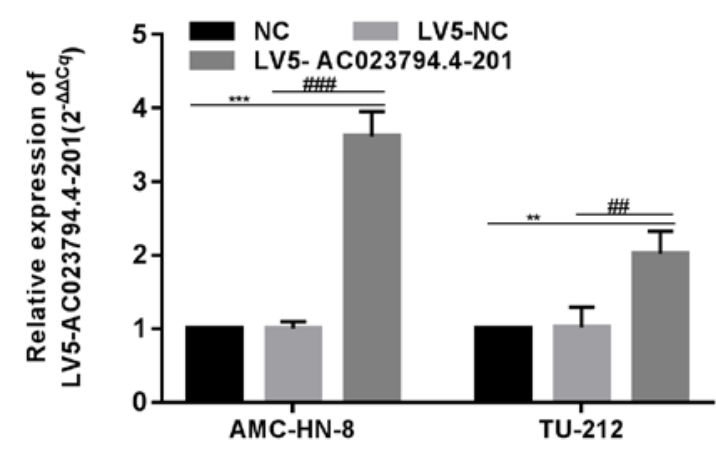

Figure 1. Transfection efficiency of AC023794.4-201 overexpression lentiviruses in TU-212 and AMC-HN8 cell lines. Expression levels of AC023794.4-201 in LSCC cell lines were determined using reverse transcription-quantitative PCR. Expression levels were quantified using the $2^{-\Delta \Delta C q}$ method (15). P-value is comparing relative expression of AC023794.4-201 in LSCC cells vs. Blank group, ${ }^{* *} \mathrm{P}<0.01,{ }^{* * *} \mathrm{P}<0.001$. P-value is comparing relative expression of AC023794.4-201 in LSCC cells vs. LV5-NC group, ${ }^{\# \#} \mathrm{P}<0.001,{ }^{\# \#} \mathrm{P}<0.001$. LSCC, laryngeal squamous cell carcinoma; LV5, lentiviral overexpression; NC, negative control.

Hematoxylin and eosin $(H \& E)$ staining. Tumor tissues were fixed in $10 \%$ neutral formalin for 3 days at $25^{\circ} \mathrm{C}$. The fixed tumor tissues were dehydrated with an ascending concentration of alcohol (Sinopharm Chemical Reagent Co., Ltd) $(75,85$ and $100 \%)$ and washed with xylene. Sections were embedded in paraffin and cut into 5- $\mu \mathrm{m}$-thick sections that were incubated at $60^{\circ} \mathrm{C}$ in an electric thermostatic blast oven for $3 \mathrm{~h}$. The following experiments are performed at room temperature. The tissue sections were subsequently deparaffinized in $20 \mathrm{ml}$ xylene, rehydrated in a descending alcohol series ( $5 \mathrm{ml} ; 100,85$ and $75 \%)$ and soaked with distilled water for $5 \mathrm{~min}$. Sections were stained with $0.3 \%$ hematoxylin for $10 \mathrm{~min}$ and subsequently differentiated in $0.5 \%$ alcohol hydrochloride and $0.25 \%$ ammonia water for $5 \mathrm{sec}$. Then, sections were stained with $0.5 \%$ eosin solution for 1 min and dehydrated with an ascending alcohol series (75, 85 and $100 \%$ ) for $5 \mathrm{~min}$. Finally, sections were cleared with xylene, mounted with neutral gum and visualized using an inverted fluorescence microscope (Nikon, Corporation; magnification, $\mathrm{x} 100$ ).

TEM. Tumors were cut into $1 \mathrm{~mm}^{3}$-thick tissue blocks and fixed with 3\% glutaraldehyde at $4^{\circ} \mathrm{C}$ for $2 \mathrm{~h}$. Tissues were rinsed with $0.1 \mathrm{M}$ phosphate rinsing solution 3 times for $15 \mathrm{~min}$, then fixed with $1 \%$ osmium tetroxide for $2 \mathrm{~h}$ and rinsed with $0.1 \mathrm{M}$ phosphate rinsing solution 3 times for $15 \mathrm{~min}$ at $4^{\circ} \mathrm{C}$. Rinsed tissue blocks were dehydrated in an ascending ethanol series $(50,70$ and $90 \%),(90 \%$ ethanol and $90 \%$ acetone at a 1:1 equivalent ratio), 90 and $100 \%$ acetone for $15 \mathrm{~min}$ at $4^{\circ} \mathrm{C}$ and subsequently dehydrated with $100 \%$ acetone at room temperature for $15 \mathrm{~min}$. The tissues were impregnated with 618 epoxy resin (Shanghai Resin Factory Co., Ltd). The embedded samples were cut into $70 \mathrm{~nm}$-thick sections with an ultrathin slicer. The sections were stained with $2 \%$ uranyl acetate for $10 \mathrm{~min}$ and $2 \%$ lead citrate for $5 \mathrm{~min}$ at room temperature. Finally, the apoptotic morphological changes of cells were visualized using a Hitachi H-7650 transmission electron microscope (Hitachi, Ltd.; magnification, x15,000). 
A

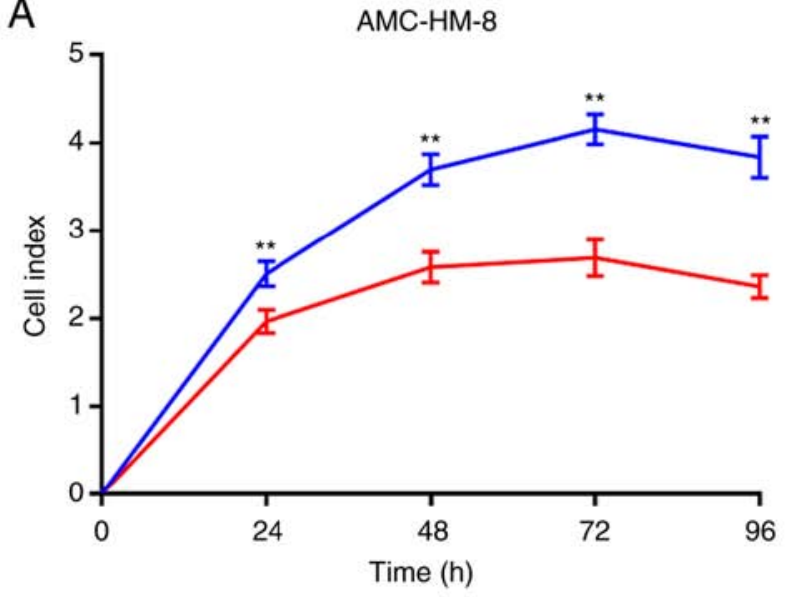

- LV5-NC

B

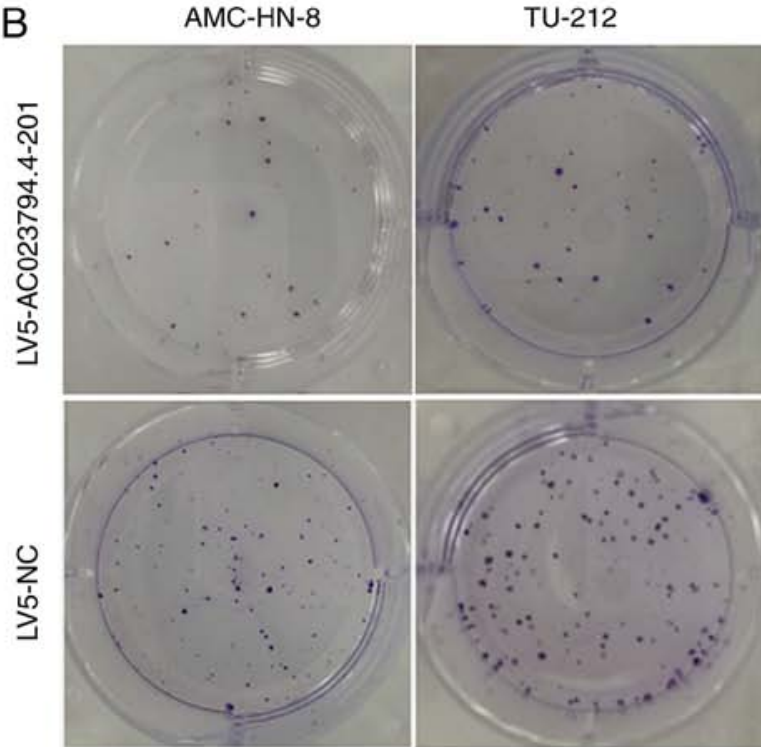

TU-212

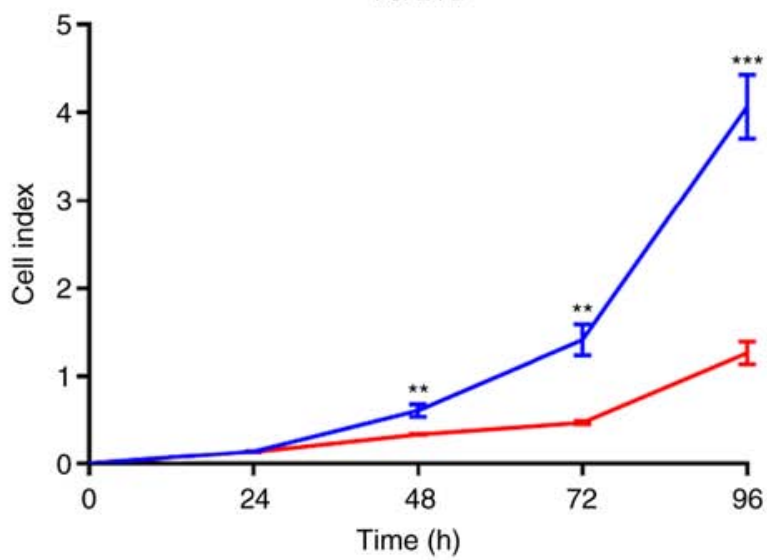

- LV5-NC

LV5-AC023794.4-201

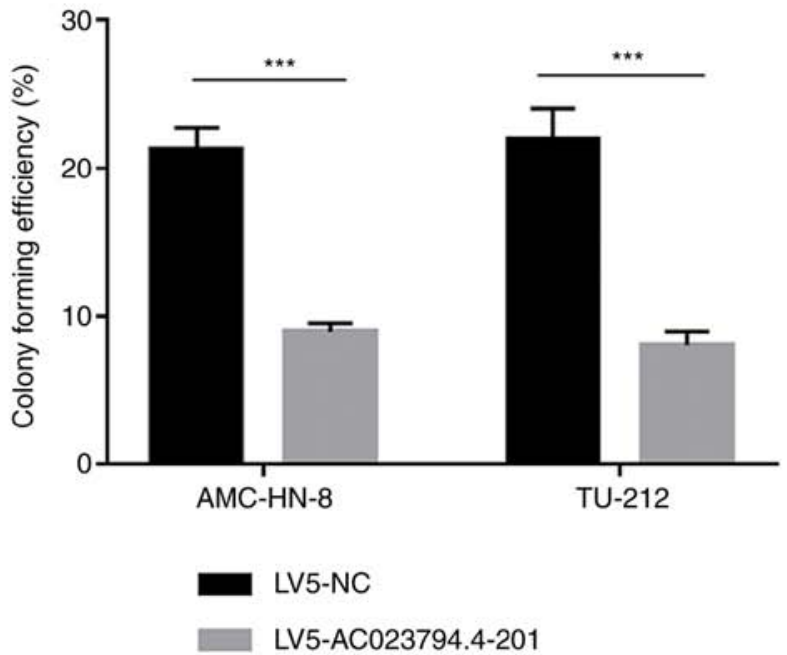

Figure 2. AC023794.4-201 inhibits laryngeal squamous cell carcinoma cell proliferation and colony formation. Cell proliferation in cell lines transfected with LV5-NC or LV5-AC023794.4-201 was determined using a (A) real-time cell analyzer assay and (B) colony formation assay. P-value is comparing cell index of 2 laryngeal squamous cell carcinoma cell lines vs. LV5-NC group. ${ }^{* *} \mathrm{P}<0.01,{ }^{* * *} \mathrm{P}<0.01$. LV5, lentiviral overexpression; NC, negative control; CI, cell index.

Statistical analysis. Cell function experiments including RTCA, cell cloning, flow cytometry, migration experiment and PCR were repeated 3 times. Statistical analysis was performed using SPSS 18.0 software (SPSS Inc.) and all quantitative data are presented as the mean \pm SD. Unpaired Student's t-tests were used to analyze the statistical differences between the groups in the cell functional assays, whereas one-way ANOVA was used to determine the statistical differences between $>2$ groups, followed by a Tukey's test for multiple comparisons. $\mathrm{P}<0.05$ was considered to indicate a statistically significant difference.

\section{Results}

Overexpression of AC023794.4-201 in LSCC cell lines. AC023794.4-201 was previously discovered to be expressed at low levels in the cancerous tissues of patients with LSCC with low differentiation, a high $\mathrm{T}$ stage and cervical lymph node metastasis (14). Thus, to assess the role of AC023794.4-201 in tumor progression, AC023794.4-201 was overexpressed in two LSCC cell lines using AC023794.4-201 overexpression lentiviruses. AC023794.4-201 expression levels in LV5-AC023794.4-201-transfected cells were significantly increased compared with the LV5-NC-transfected cells, whereas there was no significant difference observed between the NC group and LV5-NC group (Fig. 1).

AC023794.4-201 inhibits LSCC cell proliferation and colony formation. Cell proliferation following AC023794.4-201 lentiviral transfection was analyzed using a RTCA assay. The cell index of LV5-AC023794.4-201-transfected cells in both LSCC cell lines was significantly decreased compared with the LV5-NC groups (Fig. 2A). Moreover, the colony forming ability of LV5-AC023794.4-201-transfected cells was significantly decreased in both cell lines compared with the LV5-NC-transfected cells (Fig. 2B). 

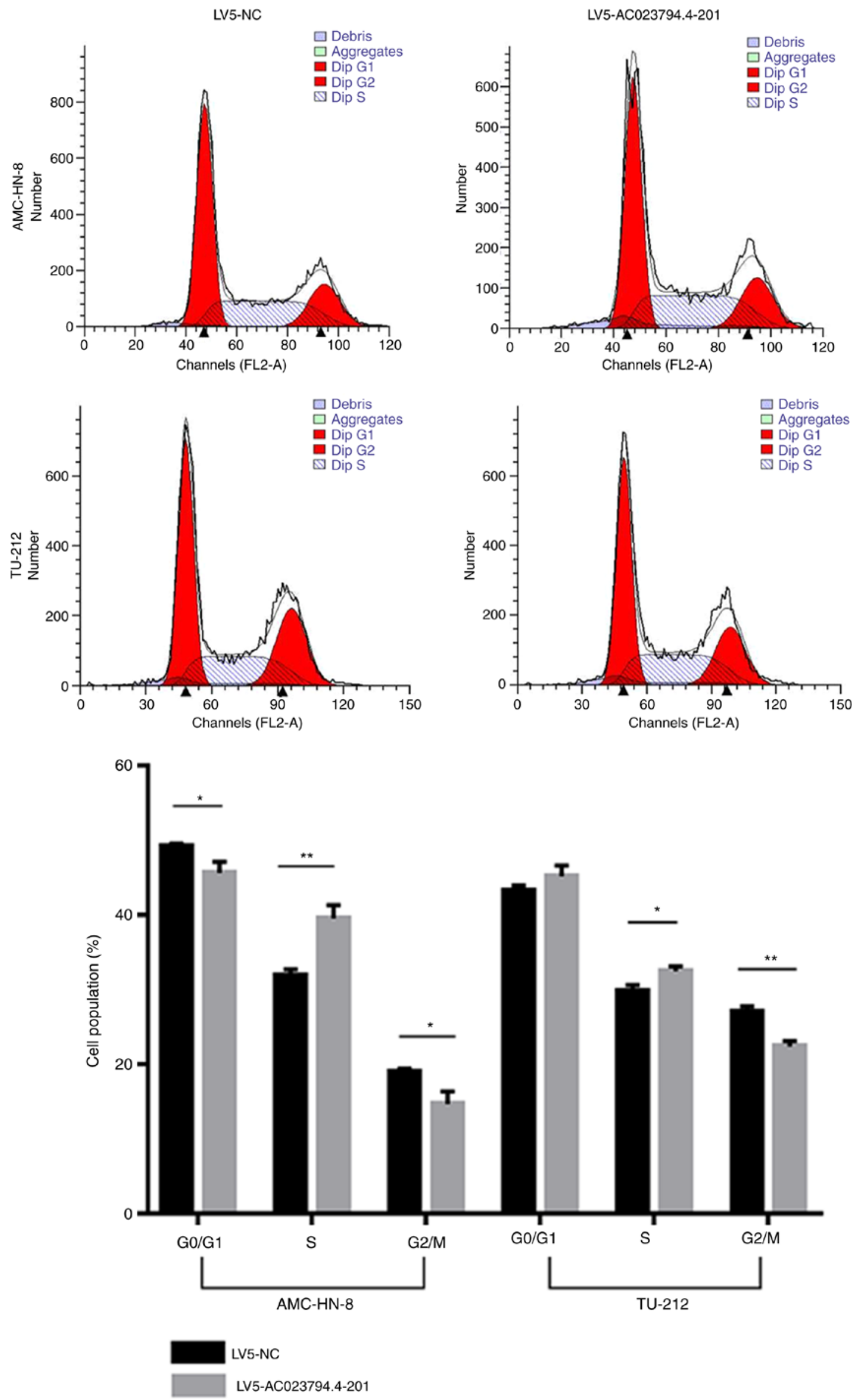

Figure 3. AC023794.4-201 arrests cell cycle progression from the $\mathrm{S}$ phase to the G2/M phase in cell lines. Cell cycle distribution was analyzed using flow cytometry in cell lines transfected with LV5-NC or LV5-AC023794.4-201. P-value is comparing proportion of cells in the cell cycle vs. LV5-NC group. " $\mathrm{P}<0.05$, ${ }^{* *} \mathrm{P}<0.01$. LV5, lentiviral overexpression; NC, negative control. 

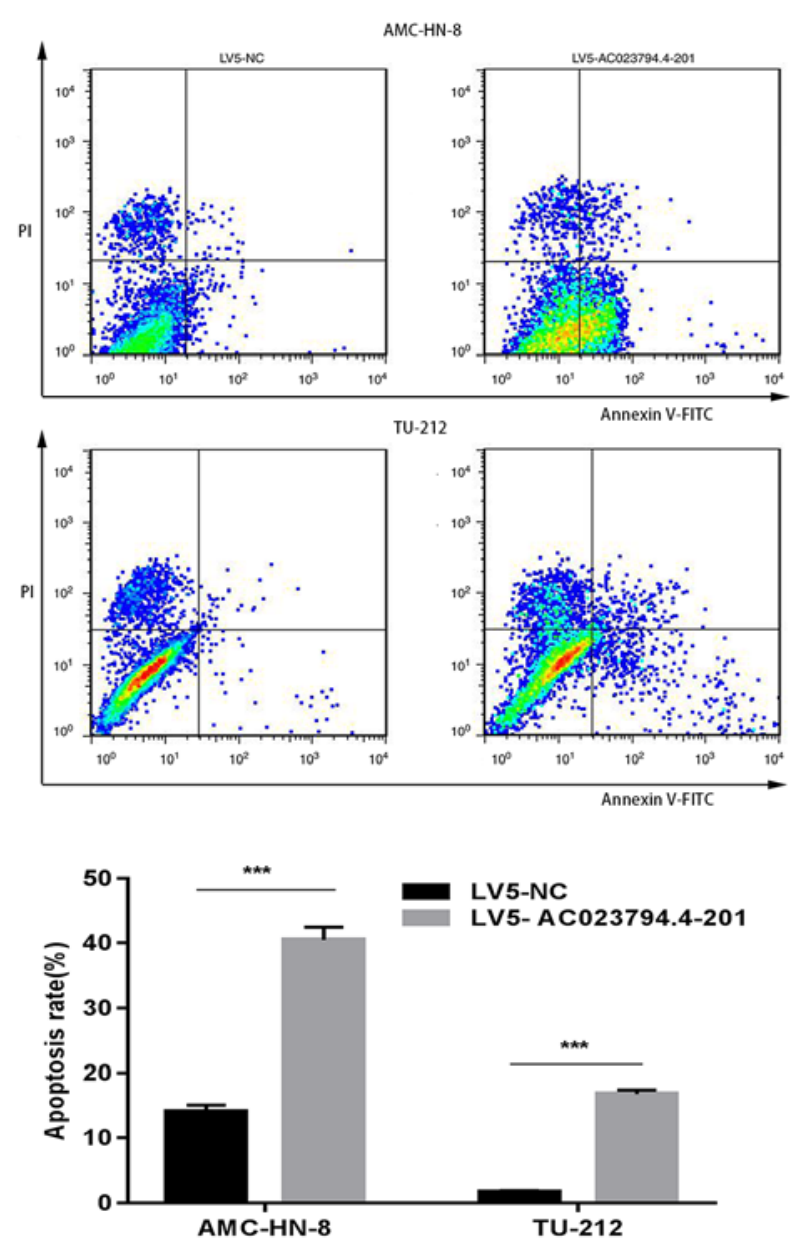

Figure 4. AC023794.4-201 can promote the apoptosis of 2 laryngeal squamous cell carcinoma cell lines. Cell apoptosis was analyzed using flow cytometry in cell lines transfected with LV5-NC or LV5-AC023794.4-201. $\mathrm{P}$-value is comparing apoptotic ratio vs. LV5-NC group. ${ }^{* * *} \mathrm{P}<0.001$. LV5, lentiviral overexpression; $\mathrm{NC}$, negative control.

Ac026166.2-001 blocks cell cycle progression from the $S$ phase to $G_{2} / M$ phase and promotes cell apoptosis. Cells in the $\mathrm{G}_{0}$ and $\mathrm{G}_{1}$ phases of the cell cycle were analyzed together as the DNA of both was the same and the 2 phases could not be distinguished from one another. Flow cytometry was used to analyze the cell cycle distribution and rate of apoptosis, and to further investigate the mechanism by which AC023794.4-201 may inhibit cell proliferation. The proportion of LV5-AC023794.4-201-transfeced cells in the $\mathrm{S}$ phase was significantly increased compared with the LV5-NC-transfected cells in both cell lines (Fig. 3). Conversely, the proportion of cells in the $\mathrm{G}_{2} / \mathrm{M}$ phase was significantly decreased in the LV5-AC023794.4-201 group compared with the LV5-C group in both cell lines (Fig. 3). Furthermore, the apoptosis assay indicated that the percentage of LV5-AC023794.4-201-transfected apoptotic cells was significantly increased compared with the LV5-NC-transfected apoptotic cells in both LSCC cell lines (Fig. 4). Thus, these finding suggest that the overexpression of AC023794.4-201 may arrest the cell cycle and promote apoptosis, thereby inhibiting cell proliferation.
Table II. Relative expression of AC023794.4-201 in xenograft.

\begin{tabular}{lcrr}
\hline Group & Number of samples & \multicolumn{1}{c}{ Mean } & Std. Deviation \\
\hline Blank & 3 & 1.1472 & 0.5811 \\
LV5-NC & 3 & 1.2413 & 0.1609 \\
High-dose & 3 & 604.4310 & 59.3730 \\
Low-dose & 3 & 85.5558 & 28.8316 \\
Total & 12 & 173.0938 & 264.8346 \\
F & 154.7410 & & \\
P-value & 0.0000 & & \\
\hline
\end{tabular}

LV5, lentiviral overexpression; NC, negative control; Std, Standard; F, F-critical value.

AC023794.4-201 inhibits LSCC cell migration. Transwell assays were used to assess the rate of cell migration in the cell lines. The results indicated that AC023794.4-201 overexpression significantly decreased the migratory ability of both LSCC cell lines compared with the LV5-NC group (Fig. 5). These findings suggest that the overexpression of AC023794.4-201 may inhibit LSCC cell proliferation, colony formation and migration.

AC023794.4-201 inhibits LSCC growth in vivo. A xenograft mouse model was constructed to further investigate whether AC023794.4-201 exerts an antitumor effect in vivo (Fig. 6A); all nude mice subcutaneously inoculated with the AMC-HN-8 cell suspension demonstrated tumor formation. Following injection of the tumors with the corresponding lentiviruses, significant differences in the expression levels of AC023794.4-201 were observed in the xenograft tumors between 4 groups $(\mathrm{P}<0.001$; Table II); the expression levels of AC023794.4-201 in the high-dose group were significantly increased compared with the other three groups ( $\mathrm{P}<0.05$ vs. blank group; ${ }^{~} \mathrm{P}<0.05$ vs. LV5-NC group; ${ }^{\wedge} \mathrm{P}<0.05$ vs. low-dose group; Fig. 6B). There was no expression difference between the low-dose group and the control group, as low concentration of LV5-AC023794.4-201 lentiviral solution has a poor transfection effect. There was no significant difference observed in the weight of mice in each group (Fig. 6C). Significant differences in the weight of xenograft tumors among the 4 groups $(\mathrm{P}<0.05$; Table III) and the weight of tumors injected with a high-dose of the AC023794.4-201 overexpression lentivirus were significantly decreased compared with the LV5-NC group ( $\mathrm{P}<0.05$; Fig. 6D). In addition, no significant differences were observed in the tumor volume of nude mice between groups on days $1,3,7,10,14,17,21$ and 24 , whereas on day 28 , significant differences were observed in the tumor volume between the 4 groups $(\mathrm{P}<0.05$; Table IV); the tumor volumes of nude mice in the high-dose AC023794.4-201 group were significantly decreased compared with the blank group $(\mathrm{P}<0.05$; Fig. $6 \mathrm{E})$. The tumor volumes of nude mice in the high-dose AC023794.4-201 group were not significantly decreased compared with the LV5-NC group.

In addition, pathological examinations of all tumor specimens were performed, alongside TEM. Following HE staining of the 4 groups of xenograft tissues, a large number of tumor 

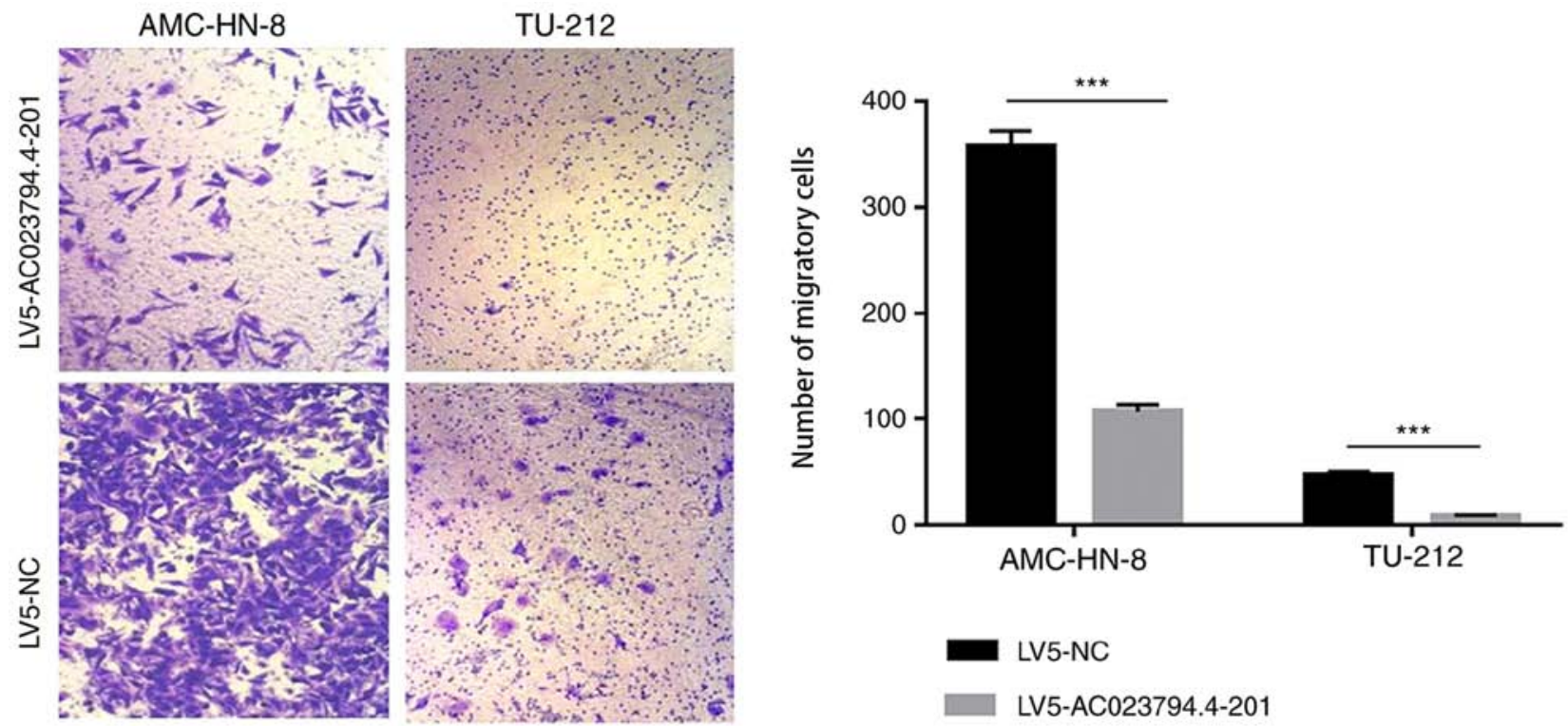

Figure 5. AC023794.4-201 inhibits laryngeal squamous cell carcinoma cell migration. The cell migratory ability was detected using a Transwell assay in cell lines transfected with LV5-NC or LV5-AC023794.4-201. Wells from TU-212 and AMC-HN8 cell lines were observed using a binocular inverted microscope and 5 fields were used to calculate the number of cells. A total of 3 biological replicates were set up and the experiment repeated 3 times. Data are presented as the mean $\pm \mathrm{SD}$. Magnification, $\mathrm{x} 40$. P-value is comparing number of migratory cells vs. LV5-NC group. ${ }^{* * *} \mathrm{P}<0.001$. LV5, lentiviral overexpression; NC, negative control.

cell clusters of various sizes and shapes with scattered small pieces or focal necrosis were observed using light microscopy which indicated that the xenograft model was successfully constructed (Fig. 7). Using transmission electron microscopy, chromatin clustered to one side of the nuclei in the high and low dose groups, which looked like a crescent or a round body indicating that the tumor cells were undergoing apoptosis. However, the tumor cells of the control groups did not exhibit these characteristics by TEM (Fig. 8). Thus, the xenograft nude mouse model revealed that AC023794.4-201 may inhibit LSCC growth in vivo.

\section{Discussion}

The development of gene sequencing and microarray techniques has facilitated the increasing number of transcript studies. Previous studies have reported that lncRNAs are closely associated with the proliferation, apoptosis, migration and invasion of LSCC cells; for example, AC026166.2-001 inhibited the proliferation and migration of LSCC cells via modulating the microRNA (miRNA/miR)-24-3p/p27 axis in cell experiments and nude mice xenograft models (16) and LOC554202 was revealed to be highly expressed in LSCC, and promoted the growth and invasion of LSCC via downregulating miR-31 in cell experiments (17). In addition, the roles of lncRNAs have been widely reported in other areas of cancer research; for instance, HOX transcript antisense RNA (HOTAIR) expression levels were discovered to be increased in oral squamous cell carcinoma (OSCC), which inhibited the expression of E-cadherin by initiating the binding of enhancer of zeste homolog 2 (EZH2) and H3K27me3 with the E-cadherin promoter, to subsequently promote tumor cell invasion and metastasis. HOTAIR may be one of critical targets in progression and metastasis in OSCC (18). Silencing
FOXD2 adjacent opposite strand RNA 1 (FOXD2-AS1) was also revealed to inhibit the growth of nasopharyngeal carcinoma (NPC) in vitro, whereas the overexpression of FOXD2-AS1 increased the growth of NPC (19). Long intergenic non-protein coding RNA regulator of reprogramming was found to be upregulated in papillary thyroid carcinoma and served an oncogenic role during thyroid cancer progression (20). HOTAIR promoted the growth of cervical cancer cells by modulating Bcl-2 via the targeting of miR-143-3p (21). Finally, maternally expressed 3 (MEG3) was revealed to be downregulated in tongue squamous cell carcinoma (TSCC), and the overexpression of MEG3 inhibited TSCC cell proliferation and promoted apoptosis (22). Thus, these findings suggested that aberrantly expressed lncRNAs may serve as tumor suppressors or oncogenes and have potential significance in the diagnosis and treatment of tumors.

AC023794.4-201, which is located on the reverse strand of chromosome $12(54,085,132-54,125,992)$, is one of the transcripts of the AC023794.4 (ENSG00000250432) gene (23). This lncRNA is 499 nucleotides in length and using a lncRNA expression microarray profile of LSCC, our previous study discovered that AC023794.4-201 expression levels were dysregulated, demonstrating increased expression levels in $86 \%(6 / 7)$ of the tumor tissues examined (24). Subsequently, 92 pairs of LSCC and corresponding paracancerous tissues were used to verify the dysregulation of AC023794.4-201 (14); however, the results revealed that AC023794.4-201 expression levels were decreased in 79\% (73/92) of the LSCC tissue samples examined. The expression levels of AC023794.4-201 in the tumor tissues of patients with Tumor (T) 3 and T4 LSCC were decreased compared with patients with T1 and T2 LSCC, and the expression levels of AC023794.4-201 in the tumor tissues of patients with lymphatic metastasis tissues (LMT) were decreased compared with patients with 

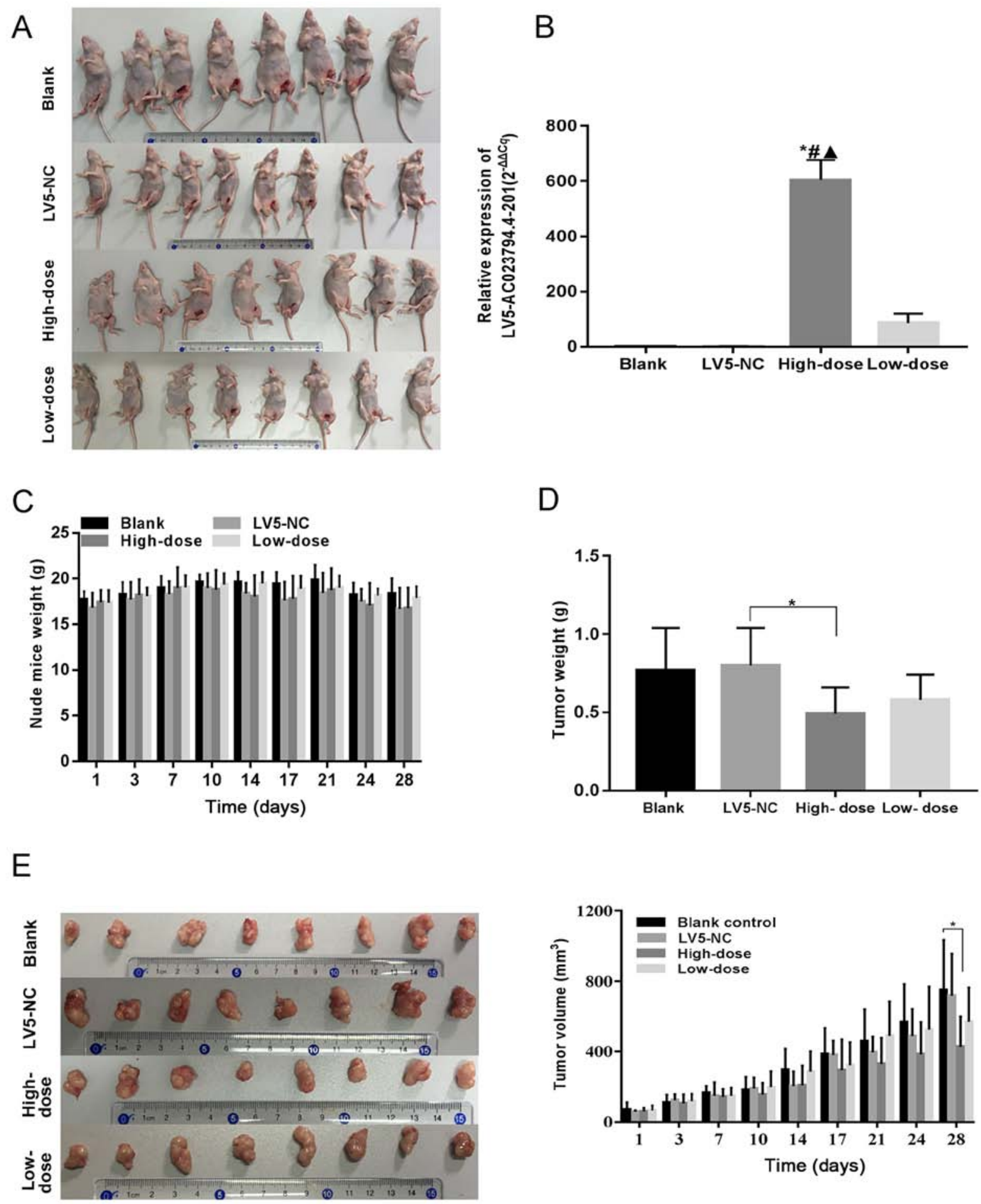

Figure 6. AC023794.4-201 inhibits laryngeal squamous cell carcinoma cell growth in nude mice. (A) In total, 32 experimental nude mice (8 mice/group) were used. (B) Expression levels of AC023794.4-201 in xenograft tumors were analyzed using reverse transcription-quantitative PCR. Relative expression levels were quantified using the $2^{-\Delta \Delta C q}$ method (13). " $\mathrm{P}$-value is comparing relative expression of AC023794.4-201 in xenografts vs. blank group, ${ }^{\circ} \mathrm{P}<0.05$; ${ }^{~} \mathrm{P}$-value is comparing relative expression of AC023794.4-201 in xenografts vs. LV5-NC group, ${ }^{\prime} \mathrm{P}<0.05$; ${ }^{\mathbf{}} \mathrm{P}$-value is comparing relative expression of AC023794.4-201 in xenografts vs. low-dose group, ${ }^{\wedge} \mathrm{P}<0.05$. (C) Weights of the nude mice in each group were not significantly different between groups. (D) Weight of nude mice tumors. P-value is comparing weight of xenografts vs. LV5-NC group, ${ }^{*} \mathrm{P}<0.05$. (E) Tumor volume of mice xenografts during the experiment. $\mathrm{P}$-value is comparing tumor volume of mice xenografts on day 28 vs. blank group, ${ }^{*} \mathrm{P}<0.05$. LV5, lentiviral overexpression; $\mathrm{NC}$, negative control.

LSCC without LMT. Furthermore, Kaplan-Meier survival curve analysis revealed that the postoperative survival rate was markedly decreased in the group expressing low levels of AC023794.4-201 compared with the group expressing high levels of AC023794.4-201 (14). These findings indicated that the results of microarray expression profiling of a small sample set can be used only as a reference, and conclusive results need to be based on large-scale PCR analysis, cell functional experiments, animal models and even further studies on the mechanisms and pathways involved. 
Table III. Tumor weight of 4 experimental groups of nude mice.

\begin{tabular}{lccc}
\hline Group & Number of samples & Mean & Std. Deviation \\
\hline Blank & 8 & 0.7738 & 0.2689 \\
LV5-NC & 8 & 0.7975 & 0.2366 \\
High-dose & 8 & 0.4875 & 0.1666 \\
Low-dose & 8 & 0.5838 & 0.1585 \\
Total & 24 & 0.6606 & 0.2414 \\
F & 3.9700 & & \\
P-value & 0.0180 & & \\
\hline
\end{tabular}

LV5, lentiviral overexpression; NC, negative control; Std, Standard; F, F-critical value.

Table IV. Volumes of xenograft tumors in 4 groups on day 28 of experiment.

\begin{tabular}{lccc}
\hline Group & Number of samples & Mean & Std. Deviation \\
\hline Blank & 8 & 750.5288 & 284.7804 \\
LV5-NC & 8 & 717.8588 & 237.3654 \\
High-dose & 8 & 428.5950 & 171.0821 \\
Low-dose & 8 & 570.4538 & 195.0623 \\
Total & 24 & 616.8591 & 251.3606 \\
F & 3.4210 & & \\
P-value & 0.0310 & & \\
\hline
\end{tabular}

LV5, lentiviral overexpression; NC, negative control; Std, Standard; F, F-critical value.

To further understand the role of AC023794.4-201 in LSCC, in the present study, AC023794.4-201 overexpression lentiviruses were used to overexpress AC023794.4-201 in LSCC cells. Cell proliferation was subsequently analyzed using a RTCA assay; AC023794.4-201 overexpression inhibited cell proliferation, with a similar result obtained from the colony formation assay, in which AC023794.4-201 upregulation inhibited colony formation. Flow cytometric analysis of the cell cycle and apoptosis was used to further investigate the role of AC023794.4-201 in LSCC. The results demonstrated that AC023794.4-201 inhibited the progression of the cell cycle from the $\mathrm{S}$ phase to the $\mathrm{G}_{2} / \mathrm{M}$ phase and promoted apoptosis. To investigate the effect of AC023794.4-201 on the migration of LSCC cells, a Transwell assay was performed and it was discovered that AC023794.4-201 overexpression inhibits the migration of LSCC cells. This result is consistent with that of our previous analysis of clinicopathological factors; that is, AC023794.4-201 levels in the tumor tissues of patients with LMT were downregulated (14). In addition, the results of the xenograft mouse model experiment further confirmed that AC023794.4-201 may serve a tumor-suppressive role in LSCC. The volume and weight of the transplanted tumors injected with the AC023794.4-201 overexpression lentivirus were significantly decreased compared with the control groups. As demonstrated using PCR, the
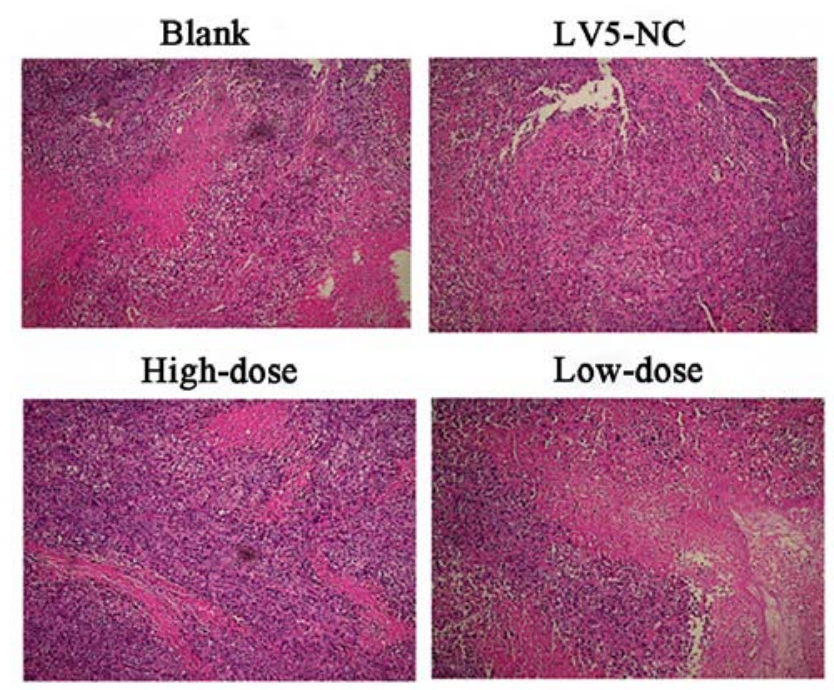

Figure 7. AC023794.4-201 inhibits LSCC growth in nude mice. Pathological examination of tumors. A large number of tumor cells of different sizes can be observed in the photographs of the 4 groups. The tumor nucleus is deeply stained with purple color. There are flaky or focal necrosis between tumor cells and the color is reddish. Magnification, x100.

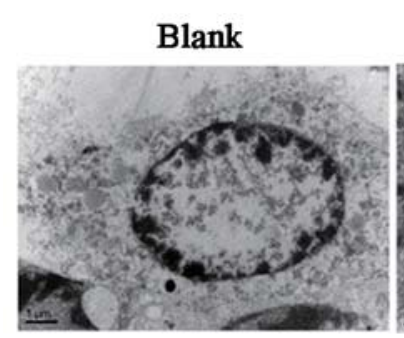

High-dose

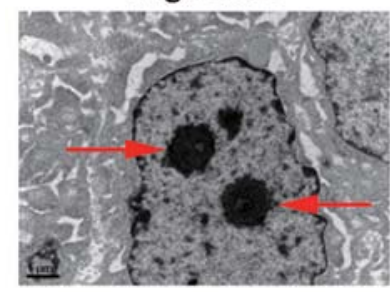

Figure 8. Transmission electron microscopy of tumors. The apoptosis of tumor tissue was observed using transmission electron microscopy (magnification, x15,000). In the high-dose AC023794.4-201 and low-dose AC023794.4-201 groups, chromatin clustered to one side of the nuclei presented by a crescent or a round body indicating that the tumor cells were undergoing apoptosis. However, the nuclei of the blank group and the LV-5-NC group were basically normal and showed no signs of apoptosis. The red arrows present aggregated chromatin. LV5, lentiviral overexpression; NC, negative control.

AC023794.40291 expression in the high-dose group was higher compared with the other 3 groups, which had a greater impact on the volume and weight of the transplanted tumor. The typical features of cell apoptosis (chromatin clustered to one side of the nuclei, appearance as a crescent or a round body) were observed. Following comprehensive analysis of the clinical data, the results of the cell functional assays and the xenograft mouse model experiment, it was concluded that AC023794.4-201 may have a tumor-suppressive function in LSCC. However, in our animal experiments, the apoptosis of tumor tissues was limited to observation by TEM, and no experiments were performed 
to investigate the expression levels of pathological markers of proliferation and apoptosis. There are numerous pathological markers of proliferation and apoptosis that have been applied to LSCC studies, such as Ki-67 and cyclin D1, which are associated with proliferation, and caspase-3 and p27 protein, which are associated with apoptosis (25-28). Thus, related immunohistochemistry experiments to assess the expression levels of these markers in vivo will be used in a follow-up experiment.

Notably, sequence alignments in the Ensembl Genes database found that Recombinant single strand-selective monofunctional uracil-DNA glycosylase 1-233 (SMUG1-223) and AC023794.4-201 had partially overlapping gene loci, and that both SMUG1-223 and AC023794.4-201 are transcribed in the same direction. SMUG1-223 is one of the transcripts of SMUG1 (ENSG00000123415), which is located on the reverse strand of chromosome 12: 54,121,277-54,173,229 (23). Previous studies have reported that SMUG1 encodes a protein that participates in base excision repair by removing uracil and certain oxidized pyrimidines from DNA, repairing oxidative damage to DNA and controlling ribosomal (r) RNA quality (29-31). AC023794.4-201 and SMUG1-223 occupy the same site (23), indicating that these two genes may interact or have similar functions. Based on these findings, it was hypothesized that AC023794.4-201 may also repair oxidative DNA damage and control rRNA quality to exert its tumor-suppressive function; however, the mechanism of action, targets, regulatory pathways and other factors involved in this hypothesis require clarification through further studies.

Numerous previous studies have discovered that lncRNAs can be used as potential diagnostic markers; Gong et al (32) performed a comparative study of serum samples from 151 patients with colorectal carcinoma and 160 healthy individuals, and found that serum hypoxia-inducible factor $1 \alpha$-antisense RNA 1(HIF1A-AS1) can serve as a latent biomarker for the diagnosis and prognosis of colorectal carcinoma. Chen et al (33) performed RT-qPCR on bladder cancer associated transcript 1 (BLACAT1) expression levels and obtained data from The Cancer Genome Atlas database, and reported that BLACAT1 expression levels may be used as a non-specific cancer diagnostic biomarker. The aforementioned study included 12 different types of cancer including: hepatocellular carcinoma, lung cancer, breast cancer, ovarian cancer, endometrial cancer, cervical cancer, prostate cancer, gastric cancer, esophagus cancer, thyroid cancer, bladder cancer and nasopharynx cancer. In addition, serum actin filament associated protein 1-antisense RNA 1 was identified as a potential diagnostic biomarker for non-small cell lung cancer (34). In fact, previous reports have demonstrated that combining multiple genetic biomarkers increased the diagnostic value of these biomarkers in cancer (35); for example, Madhavan et al (36) reported that the concomitant assessment of pancreatic cancer-initiating cells and miRNA serum-exosome marker groups significantly improved the sensitivity and specificity of pancreatic cancer diagnoses (35); and the combination of serum prostate-specific antigen (PSA) and plasma expression levels of let-7c, miR-30c, miR-141 and miR-375 were discovered to be non-invasive diagnostic biomarkers for prostate cancer screening, which were all demonstrated to be more accurate compared with PSA detection alone (37). Therefore, the identification of suitable biomarkers for the diagnosis of LSCC in combination with AC023794.4-201 represents a promising future research direction.

lncRNA AC023794.4-201 inhibits laryngeal cancer cell proliferation and promotes apoptosis in vitro and in vivo. lncRNA AC023794.4-201 plays the role of a tumor suppressor gene in the occurrence and development of LSCC. However, the specific mechanism and regulatory pathways by which lncRNA AC023794.4-201 acts needs further investigation. lncRNA AC023794.4-201 can be used as a potential diagnostic and prognostic biomarker for LSCC.

\section{Acknowledgements}

Not applicable.

\section{Funding}

This work was supported by grants from the Zhejiang Provincial Natural Science Foundation of China (grant no. LY14H160003), the Ningbo Health Branding Subject Fund (grant no. PPXK2018-02) and the Medical and Health Research Project of Zhejiang Province (grant no. 2018RC063).

\section{Availability of data and materials}

Previously reported data and the microarray profile used to support this study are available on the NCBI GEO database (accession no. GSE59652). The datasets used and/or analyzed in the current study are available from the corresponding author on reasonable request.

\section{Authors' contributions}

ZSS collected the data and analyzed the feasibility of the experiment. JY designed the experiments and the data collection strategy. QLT and WJH purchased the experimental materials and conducted the experiments. HXD and QL conducted the statistical analysis and confirmed all statistical outcomes. CCZ, YH and JX critically analyzed the data and wrote the manuscript. All authors read and approved the final manuscript.

\section{Ethics approval and consent to participate}

All animal studies were approved by and strictly followed the guidelines of the Animal Ethics and Welfare Committee of Ningbo University (approval no. AWEC-2015-10).

\section{Patient consent for publication}

Not applicable.

\section{Competing interests}

The authors declare that they have no competing interests.

\section{References}

1. Pantel $\mathrm{M}$ and Guntinas-Lichius O: Laryngeal carcinoma: Epidemiology, risk factors and survival. HNO 60: 32-40, 2012 (In German). 
2. Siegel RL, Miller KD and Jemal A: Cancer statistics, 2018. CA Cancer J Clin 68: 7-30, 2018.

3. Steuer CE, El-Deiry M, Parks JR, Higgins KA and Saba NF: An update on larynx cancer. CA Cancer J Clin 67: 31-50, 2017.

4. Forastiere AA, Ismaila N, Lewin JS, Nathan CA, Adelstein DJ, Eisbruch A, Fass G, Fisher SG, Laurie SA, Le QT, et al: Use of larynx-preservation strategies in the treatment of laryngeal cancer: American society of clinical oncology clinical practice guideline update. J Clin Oncol 36: 1143-1169, 2018.

5. Berrino F, De Angelis R, Sant M, Rosso S, Bielska-Lasota M, Coebergh JW and Santaquilani M; EUROCARE Working group: Survival for eight major cancers and all cancers combined for European adults diagnosed in 1995-99: Results of the EUROCARE-4 study. Lancet Oncol 8: 773-783, 2007.

6. Forrest ME and Khalil AM: Review: Regulation of the cancer epigenome by long non-coding RNAs. Cancer Lett 407: 106-112, 2017.

7. Quinn JJ and Chang HY: Unique features of long non-coding RNA biogenesis and function. Nat Rev Genet 17: 47-62, 2016.

8. Stein LD: Human genome: End of the beginning. Nature 431 : 915-916, 2004.

9. ENCODE Project Consortium: An integrated encyclopedia of DNA elements in the human genome. Nature 489: 57-74, 2012.

10. Huang X, Zhou X, Hu Q, Sun B, Deng M, Qi X and Lü M Advances in esophageal cancer: A new perspective on pathogenesis associated with long non-coding RNAs. Cancer Lett 413: 94-101, 2018

11. Rinn JL and Chang HY: Genome regulation by long noncoding RNAs. Annu Rev Biochem 81: 145-616, 2012.

12. Flynn RA and Chang HY: Long noncoding RNAs in cell-fate programming and reprogramming. Cell Stem Cell 14: 752-761, 2014

13. Gugnoni $M$ and Ciarrocchi A: Long non-coding RNA and epithelial-mesenchymal transition in cance. Int J Mol Sci 20 : 1924,2019

14. Tong Q, Shen Z, Li Q, Hao W and Zhou C: Expression and clinical significance of long non-coding RNA ac023794.4-201 in laryngeal squamous cell carcinoma. Chin Arch Otolaryngo Head Neck Surg 10: 564-566, 2018 (In Chinaese).

15. Livak KJ and Schmittgen TD: Analysis of relative gene expression data using real-time quantitative PCR and the 2(-Delta Delta C(T)) method. Methods 25: 402-408, 2001.

16. Shen Z, Hao W, Zhou C, Deng H, Ye D, Li Q, Lin L, Cao B and Guo J: Long non-coding RNA AC026166.2-001 inhibits cell proliferation and migration in laryngeal squamous cell carcinoma by regulating the miR-24-3p/p27 axis. Sci Rep 8: 3375, 2018

17. Yang S, Wang J, Ge W and Jiang Y: Long non-coding RNA LOC554202 promotes laryngeal squamous cell carcinoma progression through regulating miR-31. J Cell Biochem 119: 6953-6960, 2018.

18. Wu Y, Zhang L, Zhang L, Wang Y, Li H, Ren X, Wei F, Yu W, Liu $\mathrm{T}$, Wang $\mathrm{X}$, et al: Long non-coding RNA HOTAIR promotes tumor cell invasion and metastasis by recruiting EZH2 and repressing E-cadherin in oral squamous cell carcinoma. Int J Oncol 46: 2586-2594, 2015

19. Chen G, Sun W, Hua X, Zeng W and Yang L: Long non-coding RNA FOXD2-AS1 aggravates nasopharyngeal carcinoma carcinogenesis by modulating miR-363-5p/S100A 1 pathway. Gene 645: 76-84, 2018.

20. Zhang R, Hardin H, Huang W, Buehler D and Lloyd RV: Long non-coding RNA Linc-ror is upregulated in papillary thyroid carcinoma. Endocr Pathol 29: 1-8, 2018

21. Liu M, Jia J, Wang X, Liu Y, Wang C and Fan R: Long non-coding RNA HOTAIR promotes cervical cancer progression through regulating BCL2 via targeting miR-143-3p. Cancer Biol Ther 19: 391-399, 2018.

22. Jia LF, Wei SB, Gan YH, Guo Y, Gong K, Mitchelson K, Cheng J and Yu GY: Expression, regulation and roles of miR-26a and MEG3 in tongue squamous cell carcinoma. Int J Cancer 135: 2282-2293, 2014
23. Ensembl Genes. (DB/OL). https://www.ensembl.org/Homo sapiens/Gene/Summary?db=core;g=ENSG00000250432;r=12:5 4085132-54125992. Accessed June 20, 2019.

24. Shen Z, Li Q, Deng H, Lu D, Song H and Guo J: Long non-coding RNA profiling in laryngeal squamous cell carcinoma and its clinical significance: Potential biomarkers for LSCC. PLoS One 9: e108237, 2014.

25. Gioacchini FM, Alicandri-Ciufelli M, Magliulo G, Rubini C, Presutti $\mathrm{L}$ and $\mathrm{Re} \mathrm{M}$ : The clinical relevance of $\mathrm{Ki}-67$ expression in laryngeal squamous cell carcinoma. Eur Arch Otorhinolaryngol 272: 1569-1576, 2015.

26. Jovanovic IP, Radosavljevic GD, Simovic-Markovic BJ, Stojanovic SP, Stefanovic SM, Pejnovic NN and Arsenijevic NN: Clinical significance of Cyclin D1, FGF3 and p21 protein expression in laryngeal squamous cell carcinoma. J BUON 19: 944-952, 2014

27. Wang HX and Tang C: Galangin suppresses human laryngeal carcinoma via modulation of caspase-3 and AKT signaling pathways. Oncol Rep 38: 703-714, 2017

28. Yang JQ, Liang Z, Wu M, Sun YM and Liu HX: Expression of p27 and PTEN and clinical characteristics in early laryngeal squamous cell carcinoma and their correlation with recurrence. Int J Clin Exp Pathol 8: 5715-5720, 2015.

29. Jobert L, Skjeldam HK, Dalhus B, Galashevskaya A, Vågbø CB, Bjørås $\mathrm{M}$ and Nilsen $\mathrm{H}$ : The human base excision repair enzyme SMUG1 directly interacts with DKC1 and contributes to RNA quality control. Mol Cell 49: 339-345, 2013

30. Matsubara M, Tanaka T, Terato $\mathrm{H}$ and Ide $\mathrm{H}$ : Action mechanism of human SMUG1 uracil-DNA glycosylase. Nucleic Acids Symp Ser (Oxf) 49: 295-296, 2005.

31. Darwanto A, Theruvathu JA, Sowers JL, Rogstad DK, Pascal T, Goddard W III and Sowers LC: Mechanisms of base selection by human single-stranded selective monofunctional uracil-DNA glycosylase. J Biol Chem 284: 15835-15846, 2009.

32. Gong W, Tian M, Qiu H and Yang Z: Elevated serum level of lncRNA-HIF1A-AS1 as a novel diagnostic predictor for worse prognosis in colorectal carcinoma. Cancer Biomark 20: 417-424, 2017.

33. Chen X, Dai M, Zhu H, Li J, Huang Z, Liu X, Huang Y, Chen J and Dai S: Evaluation on the diagnostic and prognostic values of long non-coding RNA BLACAT1 in common types of human cancer. Mol Cancer 16: 160, 2017.

34. Li W, Li N, Kang X and Shi K: Circulating long non-coding RNA AFAP1-AS1 is a potential diagnostic biomarker for non-small cell lung cancer. Clin Chim Acta 475: 152-156, 2017.

35. Wang J, Zhou Y, Lu J, Sun Y, Xiao H, Liu M and Tian L: Combined detection of serum exosomal miR-21 and HOTAIR as diagnostic and prognostic biomarkers for laryngeal squamous cell carcinoma. Med Oncol 31: 148, 2014.

36. Madhavan B, Yue S, Galli U, Rana S, Gross W, Müller M, Giese NA, Kalthoff H, Becker T, Büchler MW and Zöller M: Combined evaluation of a panel of protein and miRNA serum-exosome biomarkers for pancreatic cancer diagnosis increases sensitivity and specificity. Int J Cancer 136: 616-627, 2015.

37. Kachakova D, Mitkova A, Popov E, Popov I, Vlahova A, Dikov T, Christova S, Mitev V, Slavov C and Kaneva R: Combinations of serum prostate-specific antigen and plasma expression levels of let-7c, miR-30c, miR-141, and miR-375 as potential better diagnostic biomarkers for prostate cancer. DNA Cell Biol 34 189-200, 2015

This work is licensed under a Creative Commons Attribution-NonCommercial-NoDerivatives 4.0 International (CC BY-NC-ND 4.0) License. 